\title{
On Consistency of Closed-loop Subspace Identifictaion with Innovation Estimation
}

Weilu Lin, S. Joe Qin, Lennart Ljung

Division of Automatic Control

E-mail: weilu.lin@gmail.com, qin@che.utexas.edu, ljung@isy.liu.se

29th June 2007

Report no.: LiTH-ISY-R-2807

Accepted for publication in Proc. Conference on Decision and Control, Bahamas 2004

Address:

Department of Electrical Engineering

Linköpings universitet

SE-581 83 Linköping, Sweden

WWW: http://WwW. control.isy.liu.se

AUTOMATIC CONTROL

REGLERTEKNIK

LINKÖPINGS UNIVERSITET

Technical reports from the Automatic Control group in Linköping are available from http://www. control.isy.liu.se/publications. 


\begin{abstract}
In this paper, we show that the consistency of closed-loop subspace identification methods (SIMs) can be achieved through innovation estimation. Based on this analysis, a sufficient condition for the consistency of a new proposed closed-loop SIM is given, A consistent estimate of th Kalman gain under closed-loop conditions is also provided based on the algorithm. A multi-input-multi-output simulation shows that it is consistent under closedloop conditions, when traditional SIMs fail to provide consistent estimates.g
\end{abstract}

Keywords: identification 


\title{
On Consistency of Closed-loop Subspace Identification with Innovation Estimation
}

\author{
Weilu Lin, S. Joe Qin*, and Lennart Ljung
}

\begin{abstract}
In this paper, we show that the consistency of closed-loop subspace identification methods (SIMs) can be achieved through innovation estimation. Based on this analysis, a sufficient condition for the consistency of a new proposed closed-loop SIM is given. A consistent estimate of the Kalman gain under closed-loop conditions is also provided based on the algorithm. A multi-input-multi-output (MIMO) simulation shows that it is consistent under closed-loop condition, when traditional SIMs fail to provide consistent estimates.
\end{abstract}

\section{INTRODUCTION}

The closed-loop identification is of special interest for a large number of engineering applications. For safety reasons or quality restrictions, it is desirable that identification experiments are carried out under the closed-loop or partial closed-loop condition. As pointed out by many researchers [1], the fundamental problem with closed-loop data is the correlation between the unmeasurable noise and the input. The closed-loop identification within prediction error methods (PEMs) framework has been extensively studied during the last twenty years, a comprehensive review in this area is provided in [2]. The advantage of PEMs is that the asymptotic variance result is available [3], which is important for "identification for control" applications [4]. A disadvantage of PEMs is that a complicated parametrization procedure is involved for multi-input-multi-output (MIMO) identification problems.

The motivation of circumventing the complicated parametrization of PEMs, especially for the MIMO identification, gave birth to subspace identification methods (SIMs), among which are canonical variate analysis (CVA) [5], N4SID [6], subspace fitting [7], and MOESP [8].

Although SIM algorithms are attractive because of the state space form that is very convenient for estimation, filtering, prediction, and control, several drawbacks have been experienced. In general, the estimates from SIMs are not as accurate as those from prediction error methods (PEMs). Further, very few SIMs are applicable to closedloop identification, even though the data satisfy identifiability conditions for traditional methods such as PEMs. In [9], we give the reasons why subspace identification approaches

*corresponding author

Supported by Weyerhaeuser Company through sponsorship of the TexasWisconsin Modeling and Control Consortium.

Weilu Lin and S. Joe Qin are with the Department of Chemical Engineering, The University of Texas at Austin, Austin, TX 78712, USA wlin@che.utexas. edu; qin@che.utexas. edu

Lennart Ljung is with the Department of Electrical Engineering, Linköping University, SE-581 83 Linköping, Sweden ljungeisy.liu.se exhibit the first drawback and propose parsimonious SIMs (PARSIMs) for open-loop applications.

Contrary to the open loop SIMs, the traditional SIMs (e.g., CVA, N4SID and MOESP) are biased under closedloop condition. Verhaegen [10] proposed a closed-loop SIM via the identification of an overall open-loop state space model followed by a model reduction step to obtain state space representations of the plant and controller. The disadvantages of the approach is that a high order overall system has to be identified, which introduces extra computational burden. Ljung and McKelvey [11] investigated the SIM through the classical realization path and proposed a recursive approach based on an ARX model as a feasible closed-loop SIM. The drawback of the approach is that the ARX parametrization is not applicable for the generic system. Recently, Chiuso and Picci [12] analyzed SIMs with feedback through stochastic realization theory and provided a theoretical analysis to construct the geometric state based on an oblique predictor space. Nevertheless, they did not provide any algorithm in detail.

To the best of our knowledge, the possibility of closedloop identification with SIMs has not been thoroughly analyzed. The main purpose of this paper is to reveal the feasibility of the consistent estimation with SIMs under the closed-loop operation. It is shown that the consistency of closed-loop SIMs can be achieved through innovation estimation.

The rest of the paper is organized as follows. In Section II, we analyze feasibility of closed-loop SIMs through innovation estimation. The consistency of closed-loop SIMs is also presented in this section. Based on this analysis, a new closed-loop SIM is presented in detail in Section III. In Section IV, a MIMO simulation is given to show the efficiency of the proposed algorithm. Section V concludes the paper.

\section{ANALYSIS OF SUBSPACE IDENTIFICATION UNDER CLOSED-LOOP CONDITION}

\section{A. Problem formulation and assumptions}

In the paper, we assume that the system to be identified can be written in innovations form as

$$
\begin{aligned}
x_{k+1} & =A x_{k}+B u_{k}+K e_{k} \\
y_{k} & =C x_{k}+D u_{k}+e_{k}
\end{aligned}
$$

where $y_{k} \in R^{n_{y}}, x_{k} \in R^{n}, u_{k} \in R^{n_{u}}$, and $e_{k} \in$ $R^{n_{y}}$ are the system output, state, input, and innovation, respectively. $A, B, C$ and $D$ are system matrices with 
appropriate dimensions. $K$ is the Kalman filter gain. The system described by Eq. 1 can also be presented as

$$
y_{k}=G(q) u_{k}+H(q) e_{k}
$$

where $G(q)=C(q I-A)^{-1} B+D$, and $H(q)=C(q I-$ $A)^{-1} K+I$. We shall assume that the input is determined through feedback as

$$
u_{k}=-F(q) y_{k}+r_{k}
$$

where $r_{k}$ is the reference signal, and $F(q)$ is the filter standing for the feedback mechanism.

To establish the statistical consistency of the SIM under closed-loop condition, we introduce following assumptions:

A1 : The system is minimal in the sense that $(C, A)$ is observable and $(A,[B, K])$ is controllable.

A2 : The innovation sequence $e_{k}$ is a stationary, zero mean, white noise process with the second order moments

$$
E\left(e_{i} e_{j}^{T}\right)=R \delta_{i j}
$$

where $\delta_{i j}$ is the Kronecker delta.

A3 : The input $u(k)$ and innovation sequence $e(j)$ are uncorrelated for $\forall j \geq k$, which implies that either the system or the controller contains a delay. To facilitate the derivation of the main results, we assume that, in this paper, the process described by Eq. 1 does not contain the direct term, i.e., $D=0$.

A4 : The reference signal and innovation sequence are uncorrelated to each other, and the reference signal is persistently exciting of a sufficient order.

A5 : The closed-loop subsystems from $r$ and $e$ to $y$ are asymptotically stable.

The closed-loop identification problem is: given a set of input/output and reference measurements, estimate the system matrices $(A, B, C, D)$, Kalman filter gain $K$ up to within a similarity transformation, and the innovation covariance matrix $R$.

Based on state space description in Eq. 1, an extended state space model can be formulated as

$$
\begin{aligned}
& Y_{f}=\Gamma_{f} X_{k}+H_{f} U_{f}+G_{f} E_{f} \\
& Y_{p}=\Gamma_{p} X_{k-p}+H_{p} U_{p}+G_{p} E_{p}
\end{aligned}
$$

where the subscripts $f$ and $p$ denote future and past horizons, respectively. The extended observability matrix is

$$
\Gamma_{f}=\left[\begin{array}{c}
C \\
C A \\
\vdots \\
C A^{f-1}
\end{array}\right]
$$

and $H_{f}$ and $G_{f}$ are Toeplitz matrices:

$$
\begin{aligned}
H_{f}= & {\left[\begin{array}{cccc}
0 & 0 & \cdots & 0 \\
C B & 0 & \cdots & 0 \\
\vdots & \vdots & \ddots & \vdots \\
C A^{f-2} B & C A^{f-3} B & \cdots & 0
\end{array}\right] } \\
G_{f}= & {\left[\begin{array}{cccc}
I & 0 & \cdots & 0 \\
C K & I & \cdots & 0 \\
\vdots & \vdots & \ddots & \vdots \\
C A^{f-2} K & C A^{f-3} K & \cdots & I
\end{array}\right] }
\end{aligned}
$$

The input and output data are arranged in the following Hankel form:

$$
\begin{aligned}
U_{f}= & {\left[\begin{array}{cccc}
u_{k} & u_{k+1} & \cdots & u_{k+N-1} \\
u_{k+1} & u_{k+2} & \cdots & u_{k+N} \\
\vdots & \vdots & \ddots & \vdots \\
u_{k+f-1} & u_{k+f} & \cdots & u_{k+f+N-2}
\end{array}\right] } \\
U_{p}= & {\left.\left[\begin{array}{cccc}
u_{k-p} & u_{k-p+1} & \cdots & u_{k-p+N-1} \\
u_{k-p+1} & u_{k-p+2} & \cdots & u_{k-p+N} \\
\vdots & \vdots & \ddots & \vdots \\
u_{k-1} & u_{k} & \cdots & u_{k+N-2}
\end{array}\right] \text { (7 }\right) }
\end{aligned}
$$

The state sequences are defined as:

$$
\begin{aligned}
X_{k} & =\left[x_{k}, x_{k+1}, \cdots, x_{k+N-1}\right] \\
X_{k-p} & =\left[x_{k-p}, x_{k-p+1}, \cdots, x_{k-p+N-1}\right]
\end{aligned}
$$

Similar formulations are made for $Y_{f}, Y_{p}, E_{f}$, and $E_{p}$. Subspace identification consists of estimating the extended observability matrix first and then the model parameters.

\section{B. Analysis of the closed-loop SIM}

The main purpose of the subsection is to explore the feasibility of closed-loop SIMs with innovation estimation. We can partition the extended state space model in Eq. 4 row-wise as follows:

$$
Y_{f}=\left[\begin{array}{c}
Y_{f 1} \\
Y_{f 2} \\
\vdots \\
Y_{f f}
\end{array}\right] ; \quad Y_{i} \triangleq\left[\begin{array}{c}
Y_{f 1} \\
Y_{f 2} \\
\vdots \\
Y_{f i}
\end{array}\right] ; i=1,2, \ldots, f
$$

Partition $U_{f}$ and $E_{f}$ in a similar way to define $U_{f i}, U_{i}, E_{f i}$, and $E_{i}$, respectively, for $i=1,2, \ldots, f$. Denote further

$$
\begin{aligned}
\Gamma_{f} & =\left[\begin{array}{c}
\Gamma_{f 1} \\
\Gamma_{f 2} \\
\vdots \\
\Gamma_{f f}
\end{array}\right] \\
H_{f i} & \triangleq\left[\begin{array}{lllll}
C A^{i-2} B & \cdots & C B & 0
\end{array}\right] \\
& \triangleq\left[\begin{array}{lllll}
H_{i-1} & \cdots & H_{1} & 0
\end{array}\right] \\
G_{f i} & \triangleq\left[\begin{array}{lllll}
C A^{i-2} K & \cdots & C K & I
\end{array}\right] \\
& \triangleq\left[\begin{array}{lllll}
G_{i-1} & \cdots & G_{1} & G_{0}
\end{array}\right]
\end{aligned}
$$


where $H_{i}$ and $G_{i}$ are the Markov parameters for the deterministic input and innovation sequence, respectively. We have the following partitioned equations:

$$
Y_{f i}=\Gamma_{f i} X_{k}+H_{f i} U_{i}+G_{f i} E_{i}
$$

for $i=1,2, \cdots, f$. Denote further,

$$
\begin{aligned}
H_{f i}^{-} & \triangleq\left[\begin{array}{lll}
H_{i-1} & \cdots & H_{1}
\end{array}\right] \\
G_{f i}^{-} & \triangleq\left[\begin{array}{lll}
G_{i-1} & \cdots & G_{1}
\end{array}\right]
\end{aligned}
$$

The partitioned $Y_{f i}$ in Eq. 11 is equal to

$$
Y_{f i}=\Gamma_{f i} X_{k}+H_{f i}^{-} U_{i-1}+G_{f i}^{-} E_{i-1}+E_{f i}
$$

By eliminating $e_{k}$ in the innovation model (Eq. 1) through iteration, it is straightforward to derive the following relation [13],

$$
X_{k}=L_{z} Z_{p}+A_{K}^{p} X_{k-p}
$$

where

$$
\begin{aligned}
& L_{z} \triangleq\left[\Delta_{p}\left(A_{K}, K\right) \Delta_{p}\left(A_{K}, B_{K}\right)\right](15 \mathrm{a}) \\
& \Delta_{p}(A, B) \triangleq\left[\begin{array}{llll}
A^{p-1} B & \cdots & A B & B
\end{array}\right] \\
& A_{K} \triangleq A-K C \\
& B_{K} \triangleq B \\
& Z_{p} \triangleq\left[\begin{array}{cc}
Y_{p}^{T} & U_{p}^{T}
\end{array}\right]^{T}
\end{aligned}
$$

Substituting this equation into Eq. 13, we obtain

$$
\begin{aligned}
Y_{f i} & =\Gamma_{f i} L_{z} Z_{p}+\Gamma_{f i} A_{K}^{p} X_{k-p} \\
& +H_{f i}^{-} U_{i-1}+G_{f i}^{-} E_{i-1}+E_{f i}
\end{aligned}
$$

for $i=1,2, \cdots, f$. Note that the second term in the right hand side (RHS) of Eq. 16 tends to zero as $p \rightarrow \infty$ if the eigenvalues of $A-K C$ are strictly inside the unit circle. Therefore, Eq. 16 reduced to

$$
Y_{f i}=\Gamma_{f i} L_{z} Z_{p}+H_{f i}^{-} U_{i-1}+G_{f i}^{-} E_{i-1}+E_{f i}
$$

Note that the future innovation, $E_{f i}$, is uncorrelated with $Z_{p}, U_{i-1}$ and $E_{i-1}$ in Eq. 17 under closed-loop condition. If the $E_{f}$ is already known, the coefficient matrices can be estimated through a straightforward linear regression as:

$$
\left[\begin{array}{ccc}
\hat{\Gamma}_{f i} L_{z} & \hat{H}_{f i}^{-} & \hat{G}_{f i}^{-}
\end{array}\right]=Y_{f i}\left[\begin{array}{c}
Z_{p} \\
U_{i-1} \\
E_{i-1}
\end{array}\right]^{\dagger}
$$

where $\dagger$ stands for pseudo-inverse operation. A remaining question is whether this approach gives us a consistent estimate of $\Gamma_{f i} L_{z}, H_{f i}^{-}$and $G_{f i}^{-}$. Here we formulate the results as follows.

Lemma 1: Under the assumptions stated at the beginning of this section, the joint input-output signal, $\chi_{k}=\left[\begin{array}{c}y_{k} \\ u_{k}\end{array}\right]$, is persistently exciting of any order.

[Proof] See Appendix A10.1 in [14].
Theorem 1: The estimates of $\Gamma_{f i} L_{z}, H_{f i}^{-}$and $G_{f i}^{-}$in Eq. 18 are consistent for $i=1,2, \cdots, f$ if and only if the joint input-output signal, $\chi_{k}=\left[\begin{array}{l}y_{k} \\ u_{k}\end{array}\right]$, is persistently exciting to the order of $p+f-1$, where $p$ and $f$ denote the past and the future horizons, respectively.

[Proof] See [15].

\section{Closed-LOOP SUBSPACE IDENTIFICATION METHODS WITH INNOVATION ESTIMATION}

From the analysis in Section II, we can conclude that, under certain assumptions, the consistency estimation with SIMs can be achieved if the innovation sequence is already known. The only challenge left now is how to estimate the innovation signal. Qin and Ljung [16] proposed an algorithm using a parsimonious model formulation with innovation estimation (PARSIM-E) for closed-loop subspace identification. Note that for the ARMAX parametrization, the extended least-squares can be applied to estimate prediction errors [14]. In this section, we introduce another algorithm (PARSIM-E1) to estimate the innovation sequence under the closed-loop condition.

\section{A. Parsimonious SIM with innovation estimation}

In this subsection, we present another parsimonious SIM algorithm with innovation estimation.

By ignoring the second term on the RHS of Eq. 16 and set $i=1$, we have

$$
Y_{f 1}=\Gamma_{f 1} L_{z} Z_{p}+E_{1}
$$

Furthermore, if we set the future horizon, $f=1$,

$$
Y_{11}=\Gamma_{11} L_{z} Z_{p}+E_{11}
$$

where $Y_{11}$ is defined in Eq. 9 and $E_{11}$ is defined in a similar way. $\Gamma_{11}$ is defined in Eq. 10a.

Therefore, a least squares estimate of the innovation process is:

$$
\hat{E}_{11}=Y_{11}-\hat{\Gamma}_{11} L_{z} Z_{p}
$$

where

$$
\hat{\Gamma}_{11} L_{z}=Y_{11} Z_{p}^{\dagger}
$$

After obtaining estimates of the innovation sequence, it is straightforward to construct $\hat{E}_{f}$ based on

$$
E_{f}=\left[\begin{array}{cccc}
e_{k} & e_{k+1} & \cdots & e_{k+N-1} \\
e_{k+1} & e_{k+2} & \cdots & e_{k+N} \\
\vdots & \vdots & \ddots & \vdots \\
e_{k+f-1} & e_{k+f} & \cdots & e_{k+f+N-2}
\end{array}\right]
$$

Now return to Eq. 17 for a general $i=1,2, \ldots, f$. Noting that

$$
E_{i}=\left[\begin{array}{c}
E_{f 1} \\
E_{f 2} \\
\vdots \\
E_{f i}
\end{array}\right]=\left[\begin{array}{c}
E_{i-1} \\
E_{f i}
\end{array}\right]
$$


and replacing $E_{i-1}$ with $\hat{E}_{i-1}$, Eq. 17 becomes

$$
Y_{f i}=\left[\begin{array}{lll}
\Gamma_{f i} L_{z} & H_{f i}^{-} & G_{f i}^{-}
\end{array}\right]\left[\begin{array}{c}
Z_{p} \\
U_{i-1} \\
\hat{E}_{i-1}
\end{array}\right]+E_{f i}
$$

The least squares estimate

$$
\left[\begin{array}{ccc}
\hat{\Gamma}_{f i} L_{z} & \hat{H}_{f i}^{-} & \hat{G}_{f i}^{-}
\end{array}\right]=Y_{f i}\left[\begin{array}{c}
Z_{p} \\
U_{i-1} \\
\hat{E}_{i-1}
\end{array}\right]^{\dagger}
$$

With the least squares estimates of $\Gamma_{f i} L_{z}$ from Eq. 26, we obtain

$$
\hat{\Gamma}_{f} L_{z}=\left[\begin{array}{c}
\hat{\Gamma}_{f 1} L_{z} \\
\hat{\Gamma}_{f 2} L_{z} \\
\vdots \\
\hat{\Gamma}_{f f} L_{z}
\end{array}\right]
$$

The observability matrix, $\Gamma_{f}$, can be estimated similarly to the order determination procedure in [9].

$$
\hat{\Gamma}_{f}=W_{1}^{-1} U_{n} S_{n}^{1 / 2}
$$

where

$$
W_{1}\left(\hat{\Gamma}_{f} L_{z}\right) W_{2}=U_{n} S_{n} V_{n}^{T}
$$

and the weighting matrices

$$
\begin{aligned}
& W_{1}=I \\
& W_{2}=\left(Z_{p} \Pi_{U_{f}}^{\perp} Z_{p}^{T}\right)^{1 / 2}
\end{aligned}
$$

Theorem 2: The estimate of $\hat{\Gamma}_{f}$ from PARSIM-E1 is consistent under the assumptions A1 to A6 stated in Section 2.1 .

[Proof] To prove the consistency of $\hat{\Gamma}_{f}$ from PARSIM-E1, it is sufficient to show that as $N \rightarrow \infty$

$$
\left[\begin{array}{lll}
\hat{\Gamma}_{f i} L_{z} & \hat{H}_{f i}^{-} & \hat{G}_{f i}^{-}
\end{array}\right] \rightarrow\left[\begin{array}{lll}
\Gamma_{f i} L_{z} & H_{f i}^{-} & G_{f i}^{-}
\end{array}\right]
$$

Note that if the innovation sequence is already known it has been proven in Theorem 1. Therefore, Theorem 2 is valid if

$$
\hat{E}_{11} \rightarrow E_{11}
$$

as $N \rightarrow \infty$, which is straightforward for a sufficient large past horizon.

\section{B. K estimation under closed-loop condition}

Di Ruscio [17] proposed a way to identify the Kalman filter gain with $\mathrm{QR}$ implementation for open loop data. It requires that $E_{f}$ is uncorrelated to $Z_{p}$ and $U_{f}$. It is invalid under closed-loop condition, if there is no delay in the controller. In this subsection, we provide a new way to calculate $K$ with closed-loop data.

Substituting the $X_{k}$ in the extended state space model (Eq. 4) with Eq. 14, we obtain

$$
Y_{f}=\Gamma_{f} L_{z} Z_{p}+\Gamma_{f} A_{K}^{p} X_{k-p}+H_{f} U_{f}+G_{f} E_{f}
$$

Omitting the second term for a sufficient large $p$, and replacing $\Gamma_{f} L_{z}$ with

$$
\hat{\Gamma}_{f} L_{z}=W_{1}^{-1} U_{n} S_{n} V_{n}^{T} W_{2}^{-1}
$$

Eq. 30 becomes

$$
\tilde{Y}_{f}=H_{f} U_{f}+G_{f} E_{f}
$$

where

$$
\tilde{Y}_{f}=Y_{f}-\hat{\Gamma}_{f} L_{z} Z_{p}
$$

We can partition $\tilde{Y}_{f}$ row-wise as follows:

$$
\tilde{Y}_{f i}=H_{f i} U_{i}+G_{f i}^{-} E_{i-1}+E_{f i} ; i=1,2, \ldots, f
$$

Again, replacing $E_{i-1}$ with $\hat{E}_{i-1}$, the least squares estimation

$$
\left[\begin{array}{cc}
\hat{H}_{f i} & \hat{G}_{f i}^{-}
\end{array}\right]=\tilde{Y}_{f i}\left[\begin{array}{c}
U_{i} \\
\hat{E}_{i-1}
\end{array}\right]^{\dagger} ; i=2,3, \ldots, f
$$

With the definition of $G_{f i}^{-}$in Eq. 12b,

$$
\hat{G}_{f i}=\left[\begin{array}{cc}
\hat{G}_{f i}^{-} & I
\end{array}\right] ; i=2,3, \ldots, f
$$

where $\hat{G}_{f i}$ is the estimation of $G_{f i}$ in Eq. 10d.

Therefore, the estimate of $G_{f}$ can be obtained based on Eq. 6b, which is lower triangular but is not exactly Toeplitz due to estimation error. After taking the average of the diagonal block components of $\hat{G}_{f}$, the Toeplitz structure of $\hat{G}_{f}$ can be preserve as,

$$
\hat{G}_{f}=\left[\begin{array}{cccc}
I & 0 & \cdots & 0 \\
\hat{G}_{1} & I & \cdots & 0 \\
\vdots & \vdots & \ddots & \vdots \\
\hat{G}_{f-1} & \hat{G}_{f-2} & \cdots & I
\end{array}\right]
$$

Furthermore, notice that

$$
\left[\begin{array}{c}
G_{1} \\
G_{2} \\
\vdots \\
G_{f-1}
\end{array}\right]=\left[\begin{array}{c}
C \\
C A \\
\vdots \\
C A^{f-2}
\end{array}\right] K=\Gamma_{f-1} K
$$

The Kalman gain, $K$, can be calculated as

$$
\hat{K}=\hat{\Gamma}_{f-1}^{\dagger}\left[\begin{array}{c}
\hat{G}_{1} \\
\hat{G}_{2} \\
\vdots \\
\hat{G}_{f-1}
\end{array}\right]
$$

where $\hat{\Gamma}_{f-1}^{\dagger}$ can be obtained as discussed in previous subsection.

After calculating the estimates of $K$, the $B$ and $D$ matrices can be estimated optimally using the estimates of A, C, $\mathrm{K}$ and $\mathrm{F}$ [18], where $F$ is the Cholesky decomposition of $\operatorname{cov}\left(\hat{E}_{11}\right)$. 


\section{Simulation EXAMPle: A MIMO PROCESS}

In this subsection, we simulate the following $2 \times 2$ linear dynamic system

$$
\begin{aligned}
& x_{k+1}=\left[\begin{array}{cccc}
0.67 & 0.67 & 0 & 0 \\
-0.67 & 0.67 & 0 & 0 \\
0 & 0 & -0.67 & -0.67 \\
0 & 0 & 0.67 & -0.67
\end{array}\right] x_{k} \\
& +\left[\begin{array}{cc}
0.6598 & -0.5256 \\
1.9698 & 0.4845 \\
4.3171 & -0.4879 \\
-2.6436 & -0.3416
\end{array}\right] u_{k} \\
& +\left[\begin{array}{cc}
-0.6968 & -0.1474 \\
0.1722 & 0.5646 \\
0.6484 & -0.4660 \\
-0.9400 & 0.1032
\end{array}\right] e_{k} \\
& y_{k}=\left[\begin{array}{cccc}
-0.3749 & 0.0751 & -0.5225 & 0.5830 \\
-0.8977 & 0.7543 & 0.1159 & 0.0982
\end{array}\right] x_{k}
\end{aligned}
$$

The output feedback controller is

$$
u_{k}=r_{k}+F_{b} y_{k}
$$

where $r_{k}$ is the reference signal and $F_{b}$ is the feedback gain matrix. In the experiment, we use the pseudo-random binary signals (PRBS) with clock period of 5 samples as the reference sequences. 4000 samples of the input and output data are generated to identify the model with $\operatorname{cov}\left(e_{k}\right)=I$ and

$$
F_{b}=\left[\begin{array}{cc}
-0.25 & 0 \\
0 & -0.25
\end{array}\right]
$$

We choose $\mathrm{p}=9, \mathrm{f}=5$ for PARSIM-E1, and run 10 independent Monte Carlo simulations.

The pole estimation results for the closed-loop experiments are shown in Fig. 1 and Fig. 2. From the results we can see that the PARSIM-E1 provide consistent estimates, while the N4SID subroutine with CVA weighting results in biased estimates.

The estimates of the frequency response for the closedloop simulations are shown in Fig. 3 and Fig. 4. We can see that the estimated frequency responses from PARSIME1 match well with that of the real system. The traditional SIM fails to provide the consistent frequency responses.

The Kalman predictor's $(A-K C)$ pole estimation from N4SID and PARSIM-E1 with the closed-loop data are shown in Fig. 5 and Fig. 6, respectively. From the figures, we can conclude that PARSIM-E1 has much better performance for the Kalman gain estimation than the N4SID routine in Matlab System Identification Toolbox.

\section{CONCLUSIONS}

In this paper, the feasibility of closed-loop subspace identification is established. It is shown that SIMs are feasible and consistent for closed-loop data with roughly the same identifiability requirements as more traditional methods such as PEMs. The key idea is that the consistent identification can be achieved through innovation estimation. Based on the analysis, a new algorithm is proposed for closed-loop identification. The simulation studies show that the algorithm is consistent under closed-loop condition, while the traditional SIMs with CVA weighting fail to provide consistent estimates.

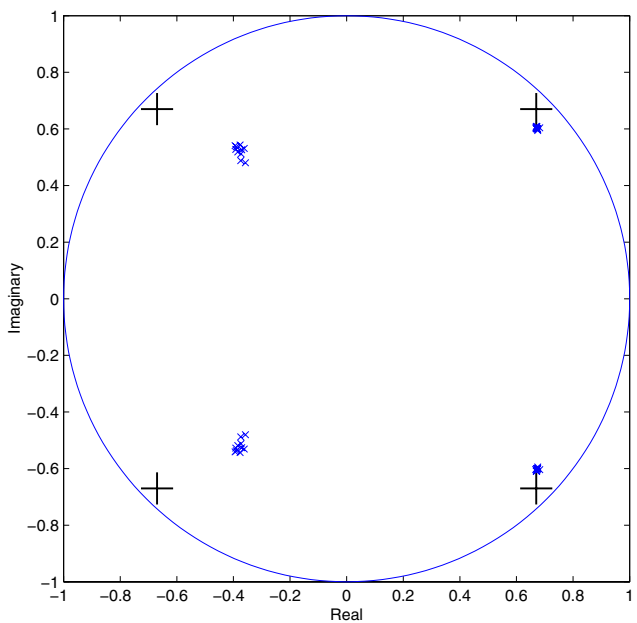

Fig. 1. The N4SID pole estimation for 10 Monte-Carlo closed-loop simulations: $\times$ estimated pole, + system pole

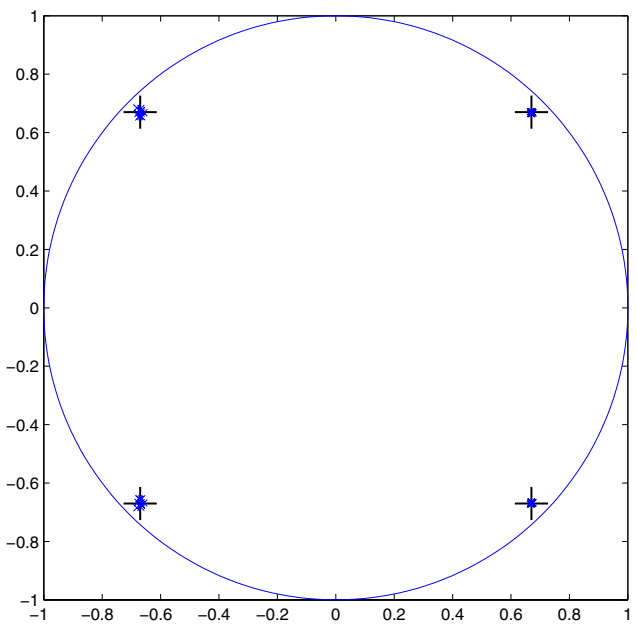

Fig. 2. The PARSIM-E1 pole estimation for 10 Monte-Carlo closed-loop simulations: $\times$ estimated pole, + system pole

\section{REFERENCES}

[1] L. Ljung, System Identification - Theory for the User, 2nd ed. Prentice Hall PTR, 1999.

[2] U. Forssell and L. Ljung, "Closed-loop identification revisited," Automatica, vol. 35, pp. 1215-1241, 1999.

[3] L. Ljung, "Asymptotic variance expression for identified black-box tranfer function models," IEEE Trans. Auto. Cont., vol. 30, pp. 834844, 1985.

[4] H. Hjalmarsson, "From experiments to closed loop control," in Proceedings of the 13th IFAC SYSID Symposium, Rotterdam, NL, Aug 2003, pp. 1-14.

[5] W. E. Larimore, "Canonical variate analysis in identification, filtering and adaptive control," in IEEE Conference on Decision and Control, Dec 1990, pp. 596-604. 


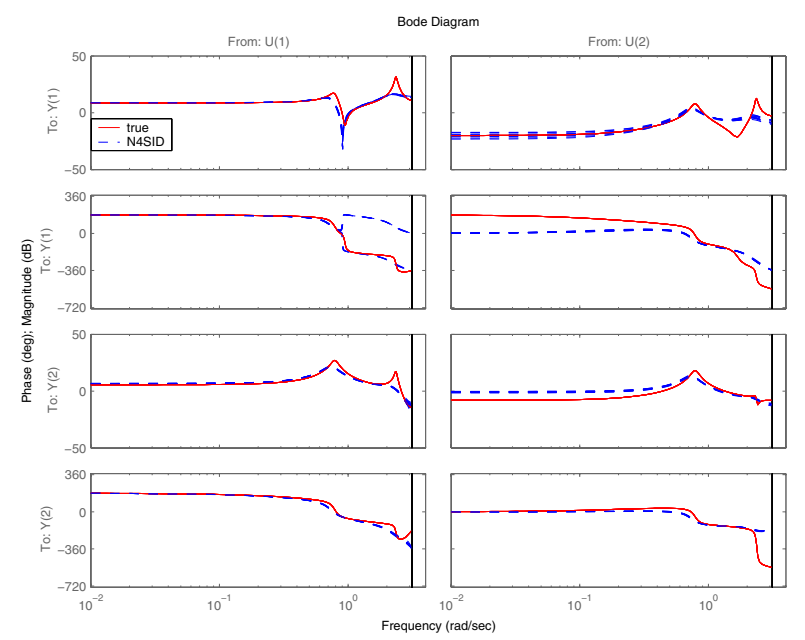

Fig. 3. The estimates of the frequency response from N4SID for MIMO closed-loop simulations

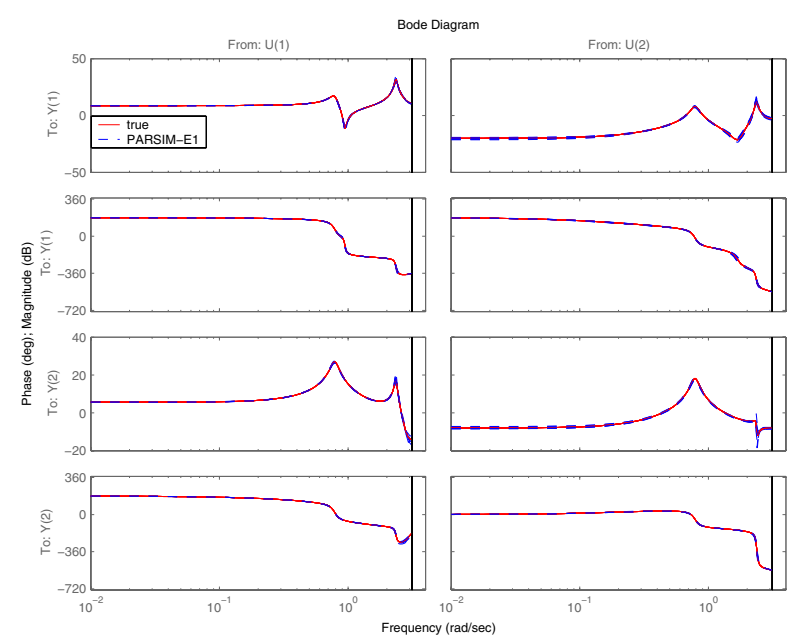

Fig. 4. The estimates of the frequency response from PARSIM-E1 for MIMO closed-loop simulations

[6] P. V. Overschee and B. D. Moor, "N4SID: subspace algorithms for the identification of combined deterministic-stochastic systems," Automatica, vol. 30, pp. 75-93, 1994.

[7] M. Jansson and B. Wahlberg, "A linear regression approach to statespace subspace system identification," Signal Processing, vol. 52, pp. 103-129, 1996.

[8] M. Verhaegen and P. Dewilde, "Subspace model identification. part I: the output-error state-space model identification class of algorithms," International Journal of Control, vol. 56, pp. 1187-1210, 1992.

[9] S. J. Qin, W. Lin, and L. Ljung, "A novel subspace identification approach with parsimonious parametrization," Submitted to Automatica, 2003.

[10] M. Verhaegen, "Application of a subspace model identification technique to identify LTI systems operating in closed-loop," Automatica, vol. 29, pp. 1027-1040, 1993.

[11] L. Ljung and T. McKelvey, "Subspace identification from closed loop data," Signal Processing, vol. 52, pp. 209-215, 1996.

[12] A. Chiuso and G. Picci, "Constructing the state of random processes with feedback," in Proceedings of the 13th IFAC SYSID Symposium, Rotterdam, NL, Aug 2003, pp. 881-886.

[13] T. Knudsen, "Consistency analysis of subspace identification methods based on linear regression approach," Automatica, vol. 37, pp. 81-89, 2001

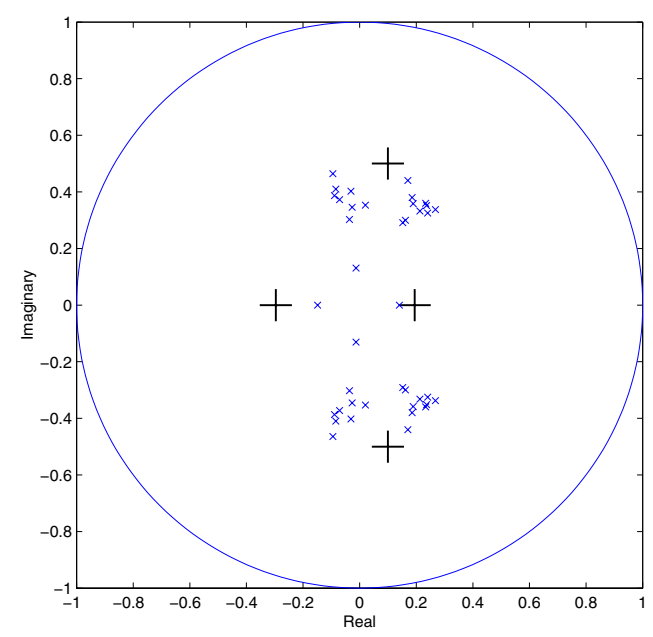

Fig. 5. The estimates of the predictor's pole from N4SID for 10 MonteCarlo MIMO closed-loop simulations: $\times$ estimated predictor's pole, + true predictor's pole

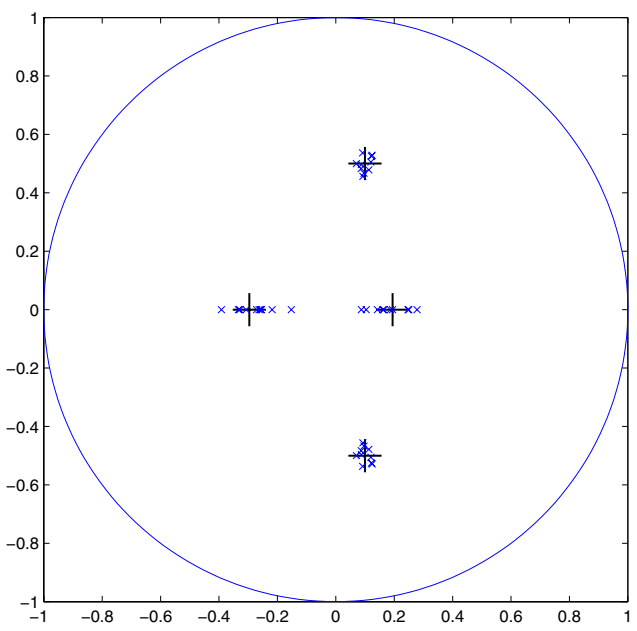

Fig. 6. The estimates of the predictor's pole from PARSIM-E1 for 10 Monte-Carlo closed-loop simulations: $\times$ estimated predictor's pole, + true predictor's pole

[14] T. Söderström and P. Soica, System Identification. Prentice Hall, 1989.

[15] W. Lin, S. J. Qin, and L. Ljung, "A framework for closed-loop subspace identification with innovation estimation," Submitted to Automatica, 2004.

[16] S. Qin and L. Ljung, "Closed-loop subspace identification with innovation estimation," in Proceedings of the 13th IFAC SYSID Symposium, Rotterdam, NL, Aug 2003, pp. 887-892.

[17] D. D. Ruscio, "Combined deterministic and stochastic system identification and realization: DSR-a subspace approach based on observations," Modeling, Identification and Control, vol. 17, pp. 193-230, 1996.

[18] S. Qin and L. Ljung, "Parallel QR implementation of subspace identification with parsimonious models," in Proceedings of the 13th IFAC SYSID Symposium, Rotterdam, NL, Aug 2003, pp. 1631-1636. 


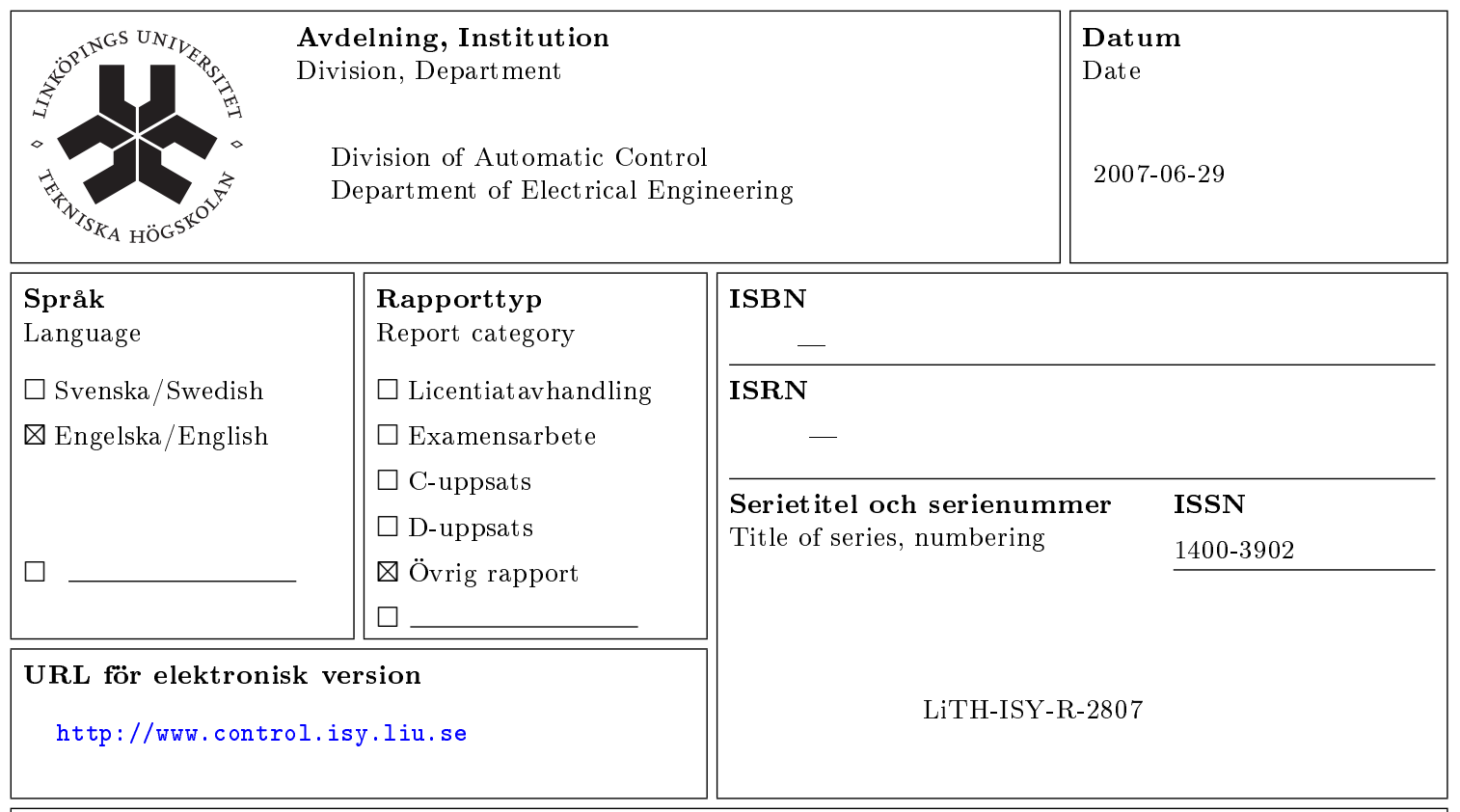

Titel On Consistency of Closed-loop Subspace Identifictaion with Innovation Estimation

Title

Författare Weilu Lin, S. Joe Qin, Lennart Ljung

Author

\section{Sammanfattning}

Abstract

In this paper, we show that the consistency of closed-loop subspace identification methods (SIMs) can be achieved through innovation estimation. Based on this analysis, a sufficient condition for the consistency of a new proposed closed-loop SIM is given, A consistent estimate of th Kalman gain under closed-loop conditions is also provided based on the algorithm. A multi-input-multi-output simulation shows that it is consistent under closed-loop conditions, when traditional SIMs fail to provide consistent estimates.g 